\title{
Recent advances and future directions in multimedia and mobile computing
}

\author{
Jong Hyuk Park • Oscar C. Au • Mikael Wiberg • \\ Changhoon Lee
}

Published online: 15 September 2011

(C) Springer Science+Business Media, LLC 2011

\section{Introduction}

Recent advances in pervasive computers, networks, telecommunication, and information technology, along with the proliferation of multimedia-capable mobile devices, such as laptops, portable media players, personal digital assistants, and cellular telephones, have stimulated the development of intelligent and pervasive multimedia applications in a ubiquitous environment. Due to constant changing environments, Mobile multimedia implies considerable challenges to operators, infrastructure builders in terms of ensuring fast, reliable services and accommodating the quick growing global customer needs. This special issue aims to foster the dissemination of high quality research in any new theory and technique and to present future directions regarding multimedia and mobile computing. This special issue welcomes papers focused on all topics ranging in the wide spectrum of multimedia and mobile computing with emphasis on theoretical aspects as well as practical frameworks and implementations. Overall, the special issue

\section{J. H. Park}

Seoul National University of Science and Technology, Seoul, Korea e-mail: parkjonghyuk1@hotmail.com

O. C. $\mathrm{Au}$

Hong Kong University of Science and Technology Hong Kong, Hong Kong, China e-mail: eeau@ust.hk

M. Wiberg

Umeå University, Umeå, Sweden

e-mail: mwiberg@informatik.umu.se

C. Lee $(\square)$

Hanshin University, Yangsan-dong, Osan, Korea

e-mail: cryptography1@gmail.com 
will outline the recent trends, major challenges, and perspectives related to multimedia and mobile computing.

We have received many manuscripts. Eleven manuscripts were finally selected for this special issue. Each manuscript selected was blindly reviewed by at least three reviewers consisting of guest editors and external reviewers. In the following section, we present a brief overview of each manuscript.

\section{Related works}

The first paper entitled "Implementation Strategies for Efficient Media Fragment Retrieval," by Wim Van Lancker et al. [11] propose and evaluate a number of implementation strategies for W3C Media Fragments URIs, which enable people to identify, share, link, and consume media fragments in a standardized way. Additionally, two optimized implementation strategies are introduced: a Media Fragment Translation Service allowing to keep existing Web infrastructure and a fully integrated Media Fragments URI server that is independent of underlying media formats.

The second paper entitled "A Middleware for a Tabletop Procedure-aware Information Display," by Kaori Fujinami et al. [4] proposes a technique to project information near a tabletop object based on a current phase in a procedure and a middleware that allows a developer to focus on editing a set of presentation rules. The middleware is then evaluated through the developments of two application scenarios.

Another paper in this special issue, entitled "User-Selectable Interactive Recommendation System in Mobile Environment," by Jung-Min Oh et al. [9] present a userselectable recommendation system which considers user's information preferences and demographics by employing collaborative filtering in a mobile environment. The goal of the paper is to provide more interactive recommendation system by adapting the optional filter for search results, and to show the prototype of flexible list of recommendation by selected option based on iPhone and Android phone environment. The cases by selected options are fifteen depend on the user's similar group. The performance parameter considered in the analysis is the Mean Absolute Error to evaluate the user-selectable recommendation system.

The fourth paper entitled "Intelligent Video Surveillance System: 3-tier contextaware surveillance system with metadata," by Yunyoung Nam et al. [8] presented an intelligent video surveillance system with the metadata rule for the exchange of analyzed information that consists of low-level context-aware, high-level context-aware and intelligent services to generate metadata for the surveillance systems. The system provides intelligent services to track moving objects in Fields Of View (FOVs) and to recognize human activities. Also, the system supports real-time moving objects tracking with Panning, Tilting and Zooming (PTZ) cameras in overlapping and non-overlapping FOV.

The fifth paper entitled "Investigating the Influence of QoS on Personal Evaluation Behaviour in a Mobile Context," by Toon De Pessemier et al. [3] is based on user feedback evaluating the content to calculate personal suggestions for the end-user. Personal preferences regarding the content are extracted from this user feedback, thereby creating user profiles. This research proves that the user feedback regarding the content might be influenced by the audiovisual quality and QoS of mobile video. As a result, 
recommender systems may generate less accurate recommendations if the QoS is variable. This paper proposes a model for correcting the user feedback by taking the audiovisual quality and QoS of the content into account during the recommendation process.

Next paper entitled "Performance of TZCD-MBOK Watermarking Scheme in T-DMB Systems," by Jung Nam Bae et al. [2] deals with T-ZCD MBOK-based watermarking techniques used in disaster broadcasting in T-DMB systems and presents the usability of the proposed techniques by simulation.

The seventh paper entitled "Evaluating Two Implementations of the Component Responsible for Decoding Video and Audio in the Brazilian Digital TV Middleware," by Tiago H. Trojahn et al. [10] investigates two Media Processing implementations using libVLC and Xine graphical libraries. Performance tests and results of both Media Processing implementations running in two different desktop architectures are discussed.

The eighth paper entitled "The Study on Content Based Multimedia Data Retrieval System," by Yang-hoon Kim et al. [6] has dealt with content-based retrieval system. In this study, the content-based retrieval system was implemented using color features from the image database that stores a number of images, and a retrieval is performed when the image vector values obtained by applying this color features to numerical expression for RGB color composition are passed over to DBMS through ODBC. As a result of test, the result values showed a relatively superior result to Swain's proposed method. Main feature of database used for the present thesis is the various background colors of the image. Even in case the objects in the image take the same shape, it can be processed into different images according to the background colors of the image. For further assignment, extracting more detailed color features requires a study on the technique of indexing color values separately for objects in the image and for background by separating the objects in the image from the background.

Another paper in this special issue, entitled "A Study on Block-based Recovery of Damaged Digital Forensic Evidence Image," by Eun-Gyeom Jang et al. [5] focus on such limitations of the existing forensic tools and introduces a new scheme that can recover and protect the evidence images on the disk. Specifically, evidence images are divided into blocks; linkage relations between those blocks are formed; and a meta-block is applied to restore the damaged blocks. Blocks in the damaged areas detected using CRC information are subject to a multidimensional block operation for recovery of damaged blocks and protection for evidence image.

The tenth paper entitled "An intrinsic semantic framework for recognizing image objects," by Nishat Ahmad et al. [1] proposes a new approach to find semantic meanings in visual object class structure, in line with the Gestalt laws of proximity. Micro level semantic structures are formed by line segments (arcs also approximated into line segments based on pixel deviation threshold) which are in close proximity. These structures are hierarchically combined till a semantic label can be assigned. The algorithm formulates semantic group structures, their inter-relations and represents these using a graph. Invariant geometrical properties of the groups and relations are used as vertex and edge labels. A graph model captures the inter class variability by analyzing the repetitiveness of structures and relations and uses it as a weighting factor for classification.

The last paper entitled "A Backup Path Routing for Guaranteeing Bandwidth in Mobile Ad hoc Networks for Multimedia Applications," by Kilhung Lee [7] suggests a routing and 
restoring scheme in mobile wireless ad hoc networks. A primary and a backup data path is established in an on-demand manner. After detecting a failure, a repairing procedure starts and restores the original path. The service quality is considered when making and restoring a path, and simulation results show that the proposed scheme supports the required service quality for multimedia applications.

\section{Conclusion}

We wish to thank all the authors for their great work and for considering the Springer's Multimedia Tools and Applications journal for submitting their papers. Special thanks go to the anonymous reviewers for their help and dedication in reviewing the papers and providing useful comments to the authors for their papers improvement. Special thanks to the EiC Professor Borko Furht for hosting this special issue in the prestigious Springer's Multimedia Tools and Applications journal, and for their excellent supports. We hope that this special issue will represent a timely and significant reference for future researches.

\section{References}

1. Ahmad N, An Y, Park J (2011) An intrinsic semantic framework for recognizing image objects. Multimedia Tools and Applications (MTAP), Online Published, February 08 2011. (doi:10.1007/s11042011-0739-8)

2. Bae JN, Kim JY, Kim G, Lee YT, Cha JS (2011) Performance of TZCD-MBOK Watermarking Scheme in T-DMB Systems. Multimedia Tools and Applications (MTAP), Online Published, February 162011. (doi:10.1007/s11042-011-0740-2)

3. De Pessemier T, De Moor K, Ketykó I, Joseph W, De Marez L, Martens L (2011) Investigating the Influence of QoS on Personal Evaluation Behaviour in a Mobile Context. Multimedia Tools and Applications (MTAP), Online Published, January 13 2011. (doi:10.1007/s11042-010-0712-y)

4. Fujinami K, Inagawa N, Nishijo K, Sokan A (2011) A Middleware for a Tabletop Procedure-aware Information Display. Multimedia Tools and Applications (MTAP), Online Published, February 222011. (doi:10.1007/s11042-011-0759-4)

5. Jang E-G, Koh B-S, Choi Y-R (2011) A Study on Block-based Recovery of Damaged Digital Forensic Evidence Image. Multimedia Tools and Applications (MTAP), Online Published, February 052011. (doi:10.1007/s11042-011-0738-9)

6. Kim Y-H, Kwon H-J, Kang J-G, Chang H (2011) The Study on Content Based Multimedia Data Retrieval System. Multimedia Tools and Applications (MTAP), Online Published, March 012011. (doi:10.1007/s11042-011-0758-5)

7. Lee K (2011) A Backup Path Routing for Guaranteeing Bandwidth in Mobile Ad hoc Networks for Multimedia Applications. Multimedia Tools and Applications (MTAP), Online Published, January 11 2011. (doi:10.1007/s11042-010-0699-4)

8. Nam Y, Rho S, Park JH (2010) Intelligent Video Surveillance System: 3-tier context-aware surveillance system with metadata. Multimedia Tools and Applications (MTAP), Online Published, December 22 2010. (doi:10.1007/s11042-010-0677-x)

9. Oh J-M, Moon NM (2011) User-Selectable Interactive Recommendation System in Mobile Environment. Multimedia Tools and Applications (MTAP), Online Published, February 08 2011. (doi:10.1007/s11042011-0737-x)

10. Trojahn TH, Gonçalves JL, Mattos JCB, Agostini LV, Da Rosa LS (2011) Evaluating Two Implementations of the Component Responsible for Decoding Video and Audio in the Brazilian Digital TV Middleware. Multimedia Tools and Applications (MTAP), Online Published, February 162011. (doi:10.1007/s11042-011-0753-x)

11. Van Wim L, Van Deursen D, Mannens E, Van de Walle R (2011) "Implementation Strategies for Efficient Media Fragment Retrieval," Multimedia Tools and Applications (MTAP), Online Published, March 26 2011. (doi:10.1007/s11042-011-0785-2) 


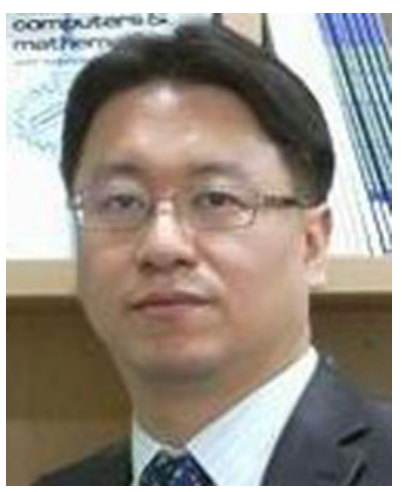

Jong Hyuk Park received his Ph.D. degree in Graduate School of Information Security from Korea University, Korea. From December, 2002 to July, 2007, Dr. Park had been a research scientist of R\&D Institute, Hanwha S\&C Co., Ltd., Korea. From September, 2007 to August, 2009, He had been a professor at the Department of Computer Science and Engineering, Kyungnam University, Korea. He is now a professor at the Department of Computer Science and Engineering, Seoul National University of Science and Technology, Korea. He has published about 100 research papers in international journals and conferences. He has been serving as chairs, program committee, or organizing committee chair for many international conferences and workshops. He is a president of Korea Information Technology Convergence Society (KITCS). He is editor-inchief (EiC) of International Journal of Information Technology, Communications and Convergence (IJITCC), InderScience. He was EiCs of the International Journal of Multimedia and Ubiquitous Engineering (IJMUE) and the International Journal of Smart Home (IJSH). He is Associate Editor/Editor of 14 international journals including 8 journals indexed by $\mathrm{SCI}(\mathrm{E})$. In addition, he has been serving as a Guest Editor for international journals by some publishers: Springer, Elsevier, John Wiley, Oxford Univ. press, Hindawi, Emerald, Inderscience. His research interests include security and digital forensics, ubiquitous and pervasive computing, context awareness, multimedia services, etc. He got the best paper award in ISA-08 conference, April, 2008. And he got the outstanding leadership awards from IEEE HPCC-09 and ISA-09, June, 2009.

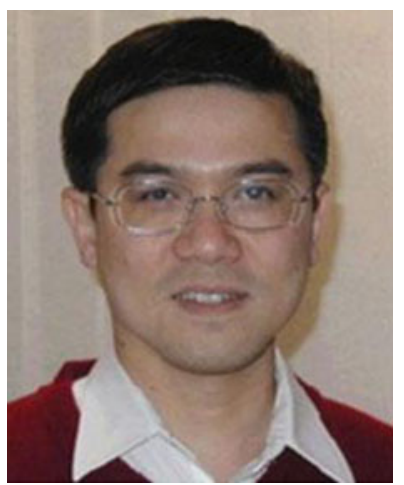

Oscar C. Au received the B.A.Sc. degree from the University of Toronto, ON, Canada, in 1986, and the M.A. and Ph.D. degrees from Princeton University, Princeton, NJ, in 1988 and 1991, respectively. After being a Postdoctoral Researcher in Princeton University for one year, he joined the Department of Electrical and Electronic Engineering, Hong Kong University of Science and Technology (HKUST), Hong Kong S.A.R., China, in 1992. $\mathrm{He}$ is now an Associate Professor, Director of the Multimedia Technology Research Center, Hong Kong, China and Advisor of the Computer Engineering Program in HKUST. His main research contributions are on video and image coding and processing, watermarking and light-weight encryption, speech and audio processing. His Research topics include fast motion estimation for MPEG-1/2/4, H.261/3/4, and AVS, optimal and fast suboptimal rate control, mode decision, transcoding, denoising, deinterlacing, post-processing, multiview coding, scalable video coding, distributed video coding, subpixel rendering, JPEG/JPEG2000, halftone image data hiding, etc. He has published/presented about 200 papers in technical journals and conferences. His fast motion estimation algorithms were incorporated into the ISO/IEC 14496-7 MPEG-4 international video coding standard and the 
China AVS-M standard. He has three U.S. patents and has applied for more than 40 patents on his signal processing techniques. He has performed forensic investigations and stood as an expert witness in the Hong Kong courts many times. He has been an Associate Editor of the IEEE TRANSACTIONS ON CIRCUITS AND SYSTEM, PART 1 and the IEEE TRANSACTIONS ON CIRCUITS AND SYSTEMS FOR VIDEO TECHNOLOGY. He is the Chairman ofMSATC, a member of the technical committee of VSPC, and a member of the technical committee of DSP of CAS. He has served on the Steering Committee of IEEE TMM and the IEEE ICME. He has also served on the Organizing Committees of the ISCAS in 1997, ICASSP in 2003, and the ISO/ IEC MPEG 71st Meeting in 2004, among others, and is in the Organizing Committee of ICIP 2010.

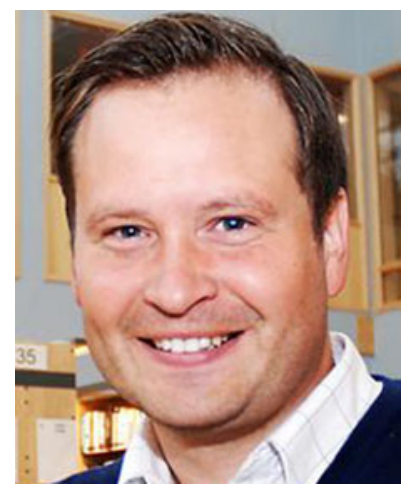

Mikael Wiberg received my Ph.D. from the Department of Informatics at Umeå University in 2001. In 2005 he became an associate professor (swe: "docent") at the same department. During the last 3 years (2008-2010) He has also served as the Research Director for Umeå Institute of Design, Umeå University, Sweden. He is a chaired professor in Human-Computer Interaction at the Department of Informatics \& Media at Uppsala University, Sweden. His research interests span across several intertwined areas including e.g. individual IT use, the emerging interaction society, empirical studies of social digitalized practices, interactive architecture, and the philosophical fundamentals of computational materials. He has served as chair, reviewer, organizer, associate editor, and session chair for a number of international conferences (e.g. full paper session chair at CHI'04). Last year, 3-5 Dec '08 he served as general conference chair for MUM'08 - 7th international conference on Mobile and Ubiquitous Multimedia in cooperation with ACM SigMobile (this year, MUM'09 is organized by Microsoft research, Nokia research and University of Cambridge, UK). Currently he is also associate editor for ISJ-Information Systems Journal.

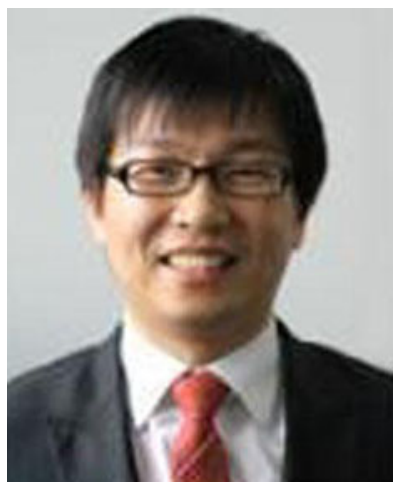

Changhoon Lee received his Ph.D. degree in Graduate School of Information Management and Security (GSIMS) from Korea University, Korea. He is now a professor in the School of Computer Engineering, Hanshin University, Korea. He is also an editorial board member of IJITCC and JIPS. Furthermore, he has been serving as a guest editor for international journals by some publishers. His research interests include information security, cryptology, embedded security in cars, digital forensics, ubiquitous and pervasive computing etc. He is currently a member of the IEEE, IEEE CS, IEEE Communications, IACR, KIISC, KIPS, KITCS, KMMS, KONI, and KIIT societies. 